\title{
Schistosoma japonicum
}

National Cancer Institute

\section{Source}

National Cancer Institute. Schistosoma japonicum. NCI Thesaurus. Code C124390.

A species of flatworms in the family Schistosomatidae. These flatworms are yellow or yellow-brown and the males are slightly larger than the other Schistosomes. This species' geographic range is restricted to China, Indonesia, and the Philippines. S. japonicum uses snails of the Oncomelania genus as its intermediate host. Within the body infestation is most frequently found in the superior mesenteric veins draining the small intestine. 